\title{
Modulation of the Contrast Response Function by Electrical Microstimulation of the Macaque Frontal Eye Field
}

\author{
Leeland B. Ekstrom, ${ }^{1,3,4}$ Pieter R. Roelfsema, ${ }^{5,6}$ John T. Arsenault, ${ }^{1,7}$ Hauke Kolster, ${ }^{1,7}$ and Wim Vanduffel ${ }^{1,2,7}$ \\ ${ }^{1}$ Athinoula A. Martinos Center for Biomedical Imaging, Massachusetts General Hospital, and 2Department of Radiology, Harvard Medical School, \\ Charlestown, Massachusetts 02129, ${ }^{3}$ Harvard-Massachusetts Institute of Technology Division of Health Sciences and Technology and ${ }^{4}$ Department of \\ Nuclear Science and Engineering, Massachusetts Institute of Technology, Cambridge, Massachusetts 02139, ${ }^{5}$ Department of Vision and Cognition, The \\ Netherlands Institute for Neuroscience, an Institute of the Royal Netherlands Academy of Arts and Sciences, 1105 BA Amsterdam, The Netherlands, \\ ${ }^{6}$ Department of Integrative Neurophysiology, Center for Neurogenomics and Cognitive Research, Vrije Universiteit, 1081 HV Amsterdam, The Netherlands, \\ and ${ }^{7}$ Laboratorium voor Neuro- en Psychofysiologie, Medical School, Campus Gasthuisberg, Katholieke Universiteit Leuven, 3000 Leuven, Belgium
}

Spatial attention influences representations in visual cortical areas as well as perception. Some models predict a contrast gain, whereas others a response or activity gain when attention is directed to a contrast-varying stimulus. Recent evidence has indicated that microstimulating the frontal eye field (FEF) can produce modulations of cortical area V4 neuronal firing rates that resemble spatial attention-like effects, and we have shown similar modulations of functional magnetic resonance imaging (fMRI) activity throughout the visual system. Here, we used fMRI in awake, fixating monkeys to first measure the response in 12 visual cortical areas to stimuli of varying luminance contrast. Next, we simultaneously microstimulated subregions of the FEF with movement fields that overlapped the stimulus locations and measured how microstimulation modulated these contrast response functions (CRFs) throughout visual cortex. In general, we found evidence for a nonproportional scaling of the CRF under these conditions, resembling a contrast gain effect. Representations of low-contrast stimuli were enhanced by stimulation of the FEF below the threshold needed to evoke saccades, whereas high-contrast stimuli were unaffected or in some areas even suppressed. Furthermore, we measured a characteristic spatial pattern of enhancement and suppression across the cortical surface, from which we propose a simple schematic of this contrast-dependent fMRI response.

\section{Introduction}

In the primate visual system, cortical sensitivity to features such as luminance contrast varies with the locus of spatial attention (Kastner and Ungerleider, 2000; Reynolds and Chelazzi, 2004). For example, attending to an isolated stimulus alters the effective luminance contrast detected by a neuron, modulating the contrast response function (CRF) of the neuron. Different models have been proposed to describe these modulations: one model suggests that attention causes a nonproportional scaling across contrasts, producing a leftward shift of the CRF or effective contrast gain (Reynolds et al., 2000; Martínez-Trujillo and Treue, 2002; Reynolds and Chelazzi, 2004). Another model proposes a proportional scaling of neuronal responses with attention, which produces either a response or activity gain effect (Williford and Maunsell, 2006). Very recent work has suggested these two mod-

Received Feb. 5, 2009; revised July 5, 2009; accepted July 15, 2009.

This work received support from a Natural Sciences and Engineering Research Council of Canada Postgraduate Scholarship, Human Frontier Science Program Organization, Geneeskundige Stichting Koningin Elisabeth, InterUniversity Attraction Pole 5/04, Excellence Financing/05/014, Geconcerteerde Onderzoeksacties 2005/18, Fonds Wetenschappelijk Onderzoek-Vlaanderen G.0.622.08, European Union Grant FP7/2007-2013 \#F2-2008-200728, National Institutes of Health Grant R01-EB000790, and National Science Foundation Grant BCS-0745436. The Martinos Center is supported by National Center for Research Resources Grant P41RR14075 and the MIND Institute. We thank H. Deng for animal training and care, G. Madan, T. van Kerkoerle, G. Bonmassar, and L. Wald for technical assistance, B. Rosen for advice and support, and C. Moore and R. Vogels for valuable comments.

Correspondence should be addressed to Dr. Wim Vanduffel, Athinoula A. Martinos Center for Biomedical Imag-

ing, 149 13th Street, Suite 2300, Charlestown, MA 02129. E-mail: wim@nmr.mgh.harvard.edu.

DOI:10.1523/JNEUROSCI.0673-09.2009

Copyright $\odot 2009$ Society for Neuroscience $\quad$ 0270-6474/09/2910683-12\$15.00/0 els may not be competitive but rather derived from a common circuit (Reynolds and Heeger, 2009), although neither offers insight into the origin of the signals causing these modulations.

Several lines of evidence suggest that the top-down sources for the presumptive feedback signals mediating spatial attention are tightly linked with the oculomotor system (Rizzolatti et al., 1987; Corbetta and Shulman, 2002; Hamker, 2005). In particular, the frontal eye field (FEF) is thought to be involved in attentiondependent modulations of sensory input. FEF would be an excellent candidate for sending top-down signals to visual cortex because of its role in visual target selection (Wardak et al., 2006), defined retinotopic map of visual space (Bruce et al., 1985), and reciprocal connections with many cortical visual areas (Huerta et al., 1987; Schall et al., 1995; Stanton et al., 1995). Experiments using electrical microstimulation of FEF (FEF-EM) have shown the following: (1) decreased contrast detection thresholds during a spatial attention task (Moore and Fallah, 2004) and changes in both (2) neuronal firing rates in cortical area V4 (Moore and Armstrong, 2003) and (3) functional magnetic resonance imaging (fMRI) activation in multiple visual areas (Ekstrom et al., 2008). Both the type of stimulus used (preferred or nonpreferred) in the single-unit study and the spatial pattern of modulation observed across the cortical surface in the fMRI study suggest that such modulations may depend on the strength of neuronal drive produced by a stimulus, similar to attentional effects on a CRF. 
To measure the effects of luminance contrast on FEF-EMinduced modulations of activity throughout visual cortex, we collected cerebral blood volume (CBV)-weighted fMRI data from two awake monkeys while simultaneously stimulating FEF. We first measured the visually driven fMRI response to stimuli of varying contrast throughout visual cortex. With the stimuli positioned in the movement fields (MFs) corresponding to the locations of chronically implanted FEF electrodes, we then measured the fMRI response to combined visual stimulation and FEF-EM and characterized how microstimulation altered each CRF. In general, we observed the largest enhancement of activity at lowest contrast, whereas high-contrast stimuli were unaffected or in some cases even suppressed by FEF-EM. These results support a contrast-gain model (Reynolds et al., 2000), and we generalize them to produce a spatial schematic of this contrast-dependent fMRI response across the cortical surface.

\section{Materials and Methods}

Many of the details of the primate imaging and stimulation techniques used have been described previously (Vanduffel et al., 2001; Nelissen et al., 2006; Ekstrom et al., 2008) and will only be summarized here briefly. All procedures were approved by the Massachusetts General Hospital Subcommittee on Research Animal Care (Protocol 2003N000338) and the Massachusetts Institute of Technology Committee on Animal Care and are in accordance with National Institutes of Health Guide for the Care and Use of Laboratory Animals.

Subject preparation. In short, we prepared two male rhesus monkeys (Macaca mulatta; MM1 and MM2, 5-7 kg, 4-5 years old) for fMRI with surgical implantation of an MR-compatible head post and then trained them for a passive fixation task. Once the subjects achieved accurate fixation performance, we chronically implanted 26 intracortical microelectrodes in the right FEF. Guided by anatomical MR images, we made a craniotomy over the right arcuate sulcus and opened the dura mater. Twenty six Teflon-coated microwires (25 $\mu \mathrm{m}$ diameter, $10 \%$ platinum/ 90\% iridium; California Fine Wire Company) were inserted by hand normal to the cortical surface (Mioche and Singer, 1988) along the rostral bank of the arcuate sulcus ( $\sim 2-6 \mathrm{~mm}$ deep). Wire tips were beveled, and $\sim 40 \mu \mathrm{m}$ of insulation was stripped before insertion. The dural and bone flaps were then replaced. We soldered the microwires to a magnetcompatible connector (Omnetics Connector Corporation), which was then encased in dental acrylic as part of the subject's headset. A ground electrode was implanted between the skull and muscle and attached to the connector. After surgery, we verified the correct anatomical placement of the electrode tips in the FEF using T2-weighted images collected at 7.0 T. We also obtained behavioral verification, in the form of successfully EM-induced saccadic eye movements, during a fixation task outside the scanner. These eye movements defined for the neurons surrounding each electrode the so-called FEF MF and the stimulation threshold to evoke a saccade (Ekstrom et al., 2008).

Anatomical and functional MRI acquisition. High-resolution, T1weighted anatomical images were collected on a $3.0 \mathrm{~T}$ full-body scanner (Trio; Siemens Healthcare) to guide electrode implantation and for the overlay of functional analyses. Under ketamine-xylazine anesthesia, a magnetization-prepared rapid-acquisition gradient echo sequence [178 sagittal slices; $256 \times 256$ in-plane matrix; repetition time (TR), $2.5 \mathrm{~s}$; echo time (TE), $4.35 \mathrm{~ms}$; inversion time, $1100 \mathrm{~ms} ; 0.35 \times 0.35 \times 0.35 \mathrm{~mm}^{3}$ isotropic voxels; flip angle, $8^{\circ}$ ] was used to obtain nine whole-brain volumes, which were averaged together to improve the signal-to-noise ratio (SNR). A single radial transmit-receive surface coil (12.5 cm diameter) was used.

Functional images were acquired on a 3.0 T full-body scanner (TIM Trio; Siemens Healthcare) with a gradient coil insert (AC88; $80 \mathrm{mT} / \mathrm{m}$ maximum gradient strength, $800 \mathrm{~T} \cdot \mathrm{m}^{-1} \cdot \mathrm{s}^{-1}$ maximum slew rate), using a gradient-echo $\mathrm{T} 2^{*}$-weighted echo-planar imaging sequence $(50$ horizontal slices; $84 \times 96$ matrix; TR, $2 \mathrm{~s}$; TE, $19 \mathrm{~ms} ; 1 \times 1 \times 1 \mathrm{~mm}^{3}$ isotropic voxels). A four-channel phased array receive coil (individual coils, $6 \mathrm{~cm}$ diameter), with generalized autocalibrating partially par- allel acquisition reconstruction (Griswold et al., 2002) and an image acceleration factor of 2, and a saddle-shaped, radial transmit-only surface coil (17 cm diameter) were used (Kolster et al., 2009).

Before each session, we injected a bolus of microcrystalline iron oxide nanoparticles (MION) $(6-10 \mathrm{mg} / \mathrm{kg})$ in isotonic sodium citrate into the femoral or saphenous vein to increase the contrast-to-noise ratio (CNR) compared with blood oxygen level-dependent (BOLD) imaging (Vanduffel et al., 2001; Leite et al., 2002). Accordingly, we have flipped the polarity of all percentage signal change values to account for the difference between MION CBV and BOLD activation maps (increased brain activation produces a decrease in MR signal in MION CBV maps).

In many cases, we were able to generate robust and well localized fMRI activation maps for a given stimulus location from a single session (Fig. $1 D$ ). We obtained a high MR CNR as a result of the use of (1) the four-channel phased array receive coil that enabled twofold image acceleration, which increased the SNR (Griswold et al., 2002) and reduced susceptibility distortions at the air-tissue interfaces around the brain, and (2) the gradient insert coil, which increased the strength and slew rate of the gradients of the MR scanner to permit shorter TR and TE values in our scanning sequence, so that we could collect more data per unit time. A shorter TE value also reduced the overall $\mathrm{T} 2{ }^{*}$ decay caused by the MION contrast agent, yielding additional increases in image SNR (Mandeville et al., 2004).

We limited our design to the five contrast values shown in Figure $1 \mathrm{~A}$ (contrast levels of $0,3,6,12$, and $50 \%$ ), chosen to provide a general overview of the contrast response function. The instances in which multiple sessions were needed for a given stimulus were primarily attributable to monkey behavior because of the long runs required by our block stimulus design: five different luminance contrasts $\times$ two electrical stimulation conditions (with and without EM stimulation) $\times$ two repetitions yielded individual runs of 20 epochs, each $24 \mathrm{~s}$ long (12 TR), resulting in runs of $480 \mathrm{~s}$. In total, 24,960 functional volumes were collected from MM1 (six sessions) and 23,280 functional volumes from MM2 (six sessions), of which 15,360 and 18,600 volumes, respectively, were used to generate the data shown.

Visual and electrical stimulation. Visual stimulation was presented to the subjects at $1024 \times 768$ resolution and $60 \mathrm{~Hz}$ refresh rate with a liquid crystal display projector (DLA-SX21; JVC) onto a translucent screen 51 $\mathrm{cm}$ from the animals' eyes. Before the beginning of our first experiment, we measured the luminance response of the red, green, and blue channels of the projector combined and separately with a photometer (CS-100 Chroma Meter; Minolta). From these response curves, we selected an approximately linear operating region and used the combined curve to calculate luminance values to generate stimuli at the desired contrasts. Compared with the stimuli in our previous study (Ekstrom et al., 2008), here we have discarded color information and increased the size of the stimuli to reduce the possibility of any mismatch between onscreen stimulus location and the corresponding FEF MF. Stimuli were 6 visual degrees diameter, monochromatic, square-wave, contrast-varying gratings, in which we defined contrast as the ratio between the difference and sum of luminance in the bright and dark regions (Michelson, 1927). The stimuli had a spatial frequency of 0.5 cycles $/^{\circ}$, were oriented at either 0 or $90^{\circ}$, and moved at $2 \%$ s perpendicular to the axis of orientation; mean luminance was matched to that of the uniformly gray background $\left(76.9 \mathrm{~cd} / \mathrm{m}^{2}\right)$.

Stimulus location was matched to the MF of the single electrode stimulated in that particular session (Moore and Armstrong, 2003; Ekstrom et al., 2008). Note that this single stimulus method differs from the multiple sequential stimuli and FEF sites approach used in our previous study, which produced fMRI activation maps averaged across all stimuli presented and electrodes used within a TR. Although such a sequential presentation is arguably closer to the physiological operating state of the visual system and produces very robust EM-only effects, the results when only one stimulus is used may be easier to interpret. Here, we used only a single stimulus-FEF electrode pairing in each session and concatenated the data after general linear model (GLM) analysis to calculate CRFs averaged across the stimulus-electrode pairings in both subjects. 

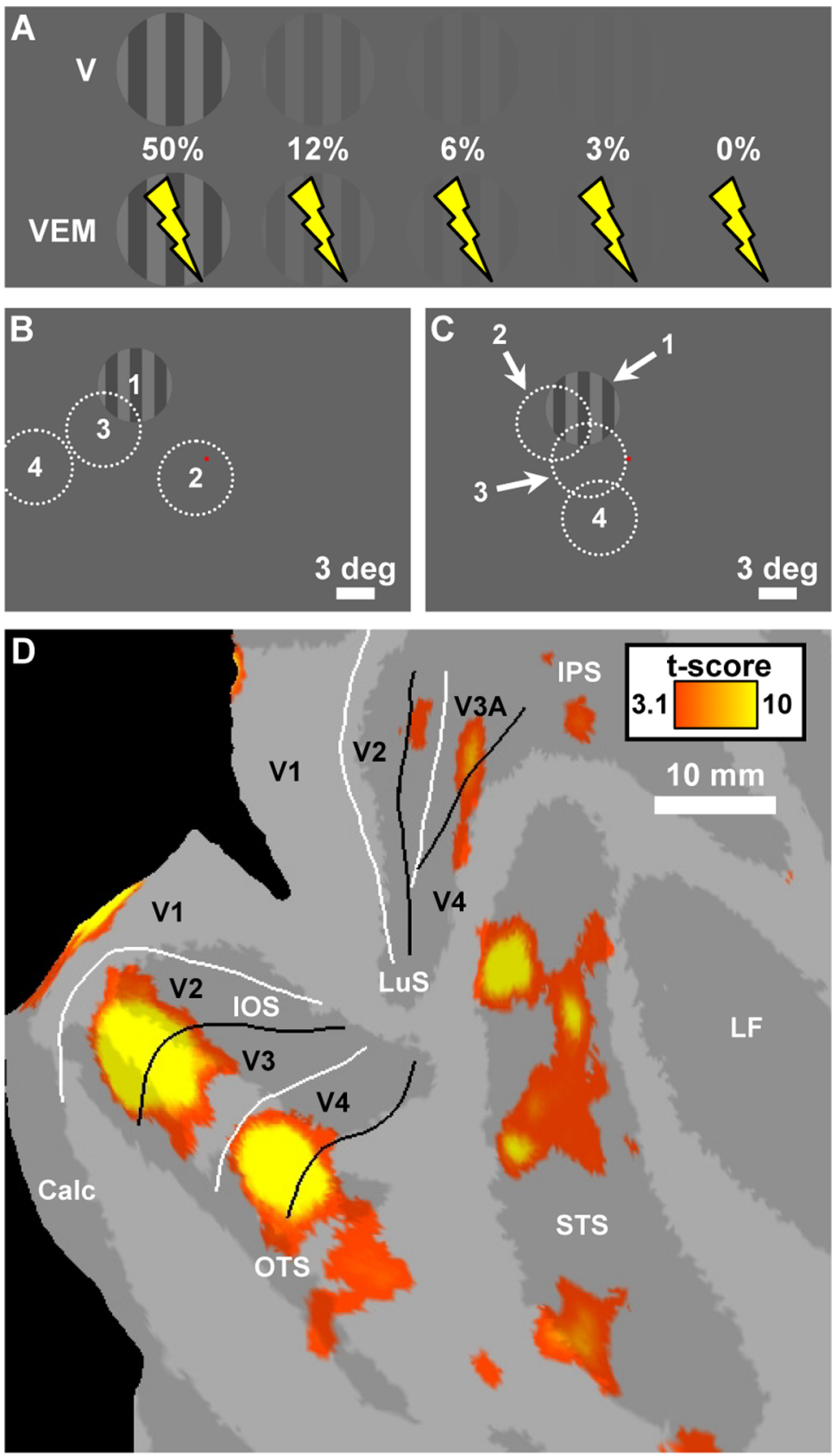

Figure 1. Stimulus conditions, stimulus locations, and localizer example. $A$, The 10 stimulus conditions used were stimuli at five different luminance contrast levels, presented without (V) and with (VEM) simultaneous FEF-EM. $0 \%$ was used as the baseline condition. The main visual effect at $50 \%$ contrast (see Materials and Methods) was used as a localizer to identify a common population of visual voxels for additional analysis. B, C, Stimulus locations for MM1 and MM2, respectively. The stimuli were centered on the endpoint of the saccade vector produced by stimulating each electrode above the threshold needed to elicit a saccade. During a given $\mathrm{fMRI}$ session, we stimulated at $50 \%$ of this behaviorally defined saccade threshold, using only one stimulus and electrode at a time. One stimulus example is shown in each panel, and the dotted white lines indicate the other stimulus positions used for that subject. The red dot in the center represents the fixation spot, which was visible in all conditions. $\boldsymbol{D}, \mathrm{A}$ thresholded $t$-score map ( $p<0.001$, uncorrected) of the localizer contrast ( $50 \%$ VEM $+50 \%$ V vs $0 \%$ VEM $+0 \%$ V) overlaid on the flattened representation of occipital cortex from MM1. The results shown correspond to the representation of stimulus 3 in $\boldsymbol{B}$. Sulci are dark gray, and white and black solid lines indicate representations of the vertical and horizontal meridians, respectively. Calc, Calcarine sulcus; CS, central sulcus; IOS, inferior occipital sulcus; IPS, intraparietal sulcus; LF, lateral fissure; LuS, lunate sulcus; OTS, occipitotemporal sulcus; deg, degree.

We used a pseudorandomized block design with multiple stimulus orders. In the epochs with FEF-EM, we stimulated the selected electrode twice within a TR (interstimulus interval of $1 \mathrm{~s}$ ). The visual stimulus was presented for $133 \mathrm{~ms}$, followed by $250 \mathrm{~ms}$ of combined visual stimulation and FEF-EM and then $617 \mathrm{~ms}$ with neither; this sequence was repeated twice within the $2 \mathrm{~s}$ TR. A central fixation point was continuously visible, and the monkeys performed a passive fixation task throughout each run. Eye position was monitored at $120 \mathrm{~Hz}$ using an infrared pupil/corneal reflection tracking system (Iscan Inc.). The EM signal was generated by an eight-channel Digital Stimulator (DS8000; World Precision Instruments), controlled by custom software that also generated the visual stimulation. Stimulation trains consisted of biphasic square-wave pulses with a frequency of $335 \mathrm{~Hz}$ for $250 \mathrm{~ms}$ and were delivered in a monopolar configuration. Each pulse consisted of $190 \mu$ s of positive and $190 \mu$ s of negative voltage, separated by $100 \mu$ s of zero voltage. The sum of the stray capacitance of the cables and the monolithic capacitor array EMI filters in the scanner penetration panel ranged from 5.1 to $5.3 \mathrm{nF}$.

Before each fMRI experiment, we determined the threshold to elicit a saccade with each electrode by varying the EM amplitude until $\sim 70 \%$ of stimulation trains induced a saccade from central fixation. A saccade vector was determined to identify the MF of the FEF site of interest for that experiment (Fig. 1, see $B$ for MM1's MFs and $C$ for MM2's MFs used in the current study); a stimulation level of 50\% of this behaviorally defined threshold was then used for the actual imaging experiment (Moore and Armstrong, 2003). At the beginning and end of each fMRI session, we measured the impedance of the stimulated channel, as well as the impedance of the three channels not used, with a $1 \mathrm{~V}, 100 \mathrm{~Hz}$ reference signal to estimate the injected current. For MM1, impedance was $32 \pm 2,35 \pm 1,48 \pm 2$, and $55 \pm$ $3 \mathrm{k} \Omega$ for the four electrodes used; for MM2, impedance was $24 \pm 1,27 \pm 1,36 \pm 1$, and $42 \pm 11 \mathrm{k} \Omega$ for the four electrodes used (mean $\pm \mathrm{SD}$ across all sessions). Estimated stimulation amplitudes for the four FEF electrodes used in MM1 were 26, 36, 55, and $72 \mu \mathrm{A}$ and in MM2 were 37, 38, 49, and $58 \mu \mathrm{A}$. We note that the current necessary to evoke saccades through chronic electrodes may be larger than with higher impedance acute electrodes. One reason for this is the possible growth of a fibrous barrier between the electrodes and neural tissue over the $2-3$ years between implantation and collection of the present dataset (Bartlett et al., 2005). Another reason may be the use here of short pulse durations (0.19 $\mathrm{ms})$ compared with those used $(0.20-0.25 \mathrm{~ms})$ in previous studies (Bruce et al., 1985).

Statistical analysis. We performed a voxelbased analysis with SPM99, following previously described procedures to fit a GLM (Friston et al., 1995; Vanduffel et al., 2001, 2002; Leite et al., 2002). Images were motion corrected within session and nonrigidly coregistered to each subject's own anatomical template using Match software (Chef d'Hotel et al., 2002). Images were then smoothed (Gaussian kernel, $\sigma=0.67 \mathrm{~mm}$ ) and registered using Match to MM1's T1-weighted anatomical volume. Global scaling and high- and low-pass filtering were used before fitting the GLM.

We used the main visual effect at $50 \%$ contrast [that is, $50 \%$ visual with FEF-EM stimulation (VEM) $+50 \%$ visual-only stimulation $(\mathrm{V})$ vs $0 \%$ 
VEM $+0 \% \mathrm{~V}]($ Fig. $1 A)$ as a localizer to identify visually driven voxels (hereafter referred to as "visual voxels") for analysis across all contrast levels. $t$-score maps of this contrast from each session in each monkey were thresholded $[p<0.05$, uncorrected; for split population analysis, low $t$ voxel distribution is 0.05 , uncorrected $>p>0.05$ corrected, high $t$ voxel distribution is $p<0.05$, corrected; correction for multiple comparisons used the Family-Wise Error procedures in SPM99 (Worsley et al., 1996)] and overlaid on the common anatomical space defined by MM1's T1-weighed anatomical volume as reconstructed by FreeSurfer (Dale et al., 1999). We created flattened cortical representations with Caret using the F99 atlas (http://www.nitrc.org/projects/caret/, http:// sumsdb.wustl.edu/sums/macaquemore.do) (Van Essen et al., 2001) (for an example of one session, see Fig. $1 D$ ). The borders of 12 visual areas were identified on this flattened cortical representation using retinotopic mapping data previously collected in three animals (Fize et al., 2003) and a Caret Atlas based on two previous studies (Ungerleider and Desimone, 1986a; Felleman and Van Essen, 1991). We extracted the population of visual voxels inside each area satisfying the above contrast and sampled the percentage change in MR signal for each condition with SPM99. The first three TRs in each epoch were excluded and an additional TR appended at the end to compensate for hemodynamic delay. SEM was calculated over the condition epochs.

To account for any head and eye movementrelated fMRI activity, covariates of no-interest from six motion realignment parameters and the $x$ and $y$ eye trace components were generated and included in the GLM analysis. Eye traces were thresholded at $\sim 2^{\circ}$, convolved with the MION hemodynamic response function, and subsampled to the TR. We performed several analyses to assess the potential effects of eye movements on fMRI activation. First, for each run collected, we aligned the stimulation events across all contrast levels to EM onset and the visual-only conditions to the matching time. We then performed a $t$ test at each time point to identify any significant differences in eye position $(p<0.05$, corrected for multiple comparisons across time points; two sample, twotailed $t$ test across all trials in that run). Any run with a significant difference at any time point was excluded from additional analysis. The mean $x$ and $y$ eye position aligned to EM onset across all sessions is illustrated in Figure 2. Second, for each subject's aggregate data, we used a one-way ANOVA to test for any significant differences in percentage fixation, saccade rate, and eye position along the $x$ and $y$ axes between the VEM and $\mathrm{V}$ conditions across all contrast levels (Table 1).

Model fitting. To the mean data points calculated in each brain area during the $\mathrm{V}$ and VEM conditions, we fit the Naka-Rushton equation (Naka and Rushton, 1966; Li et al., 2008):

$$
R=\frac{R_{\max } c^{2}}{c^{2}+c_{50}^{2}}+b
$$
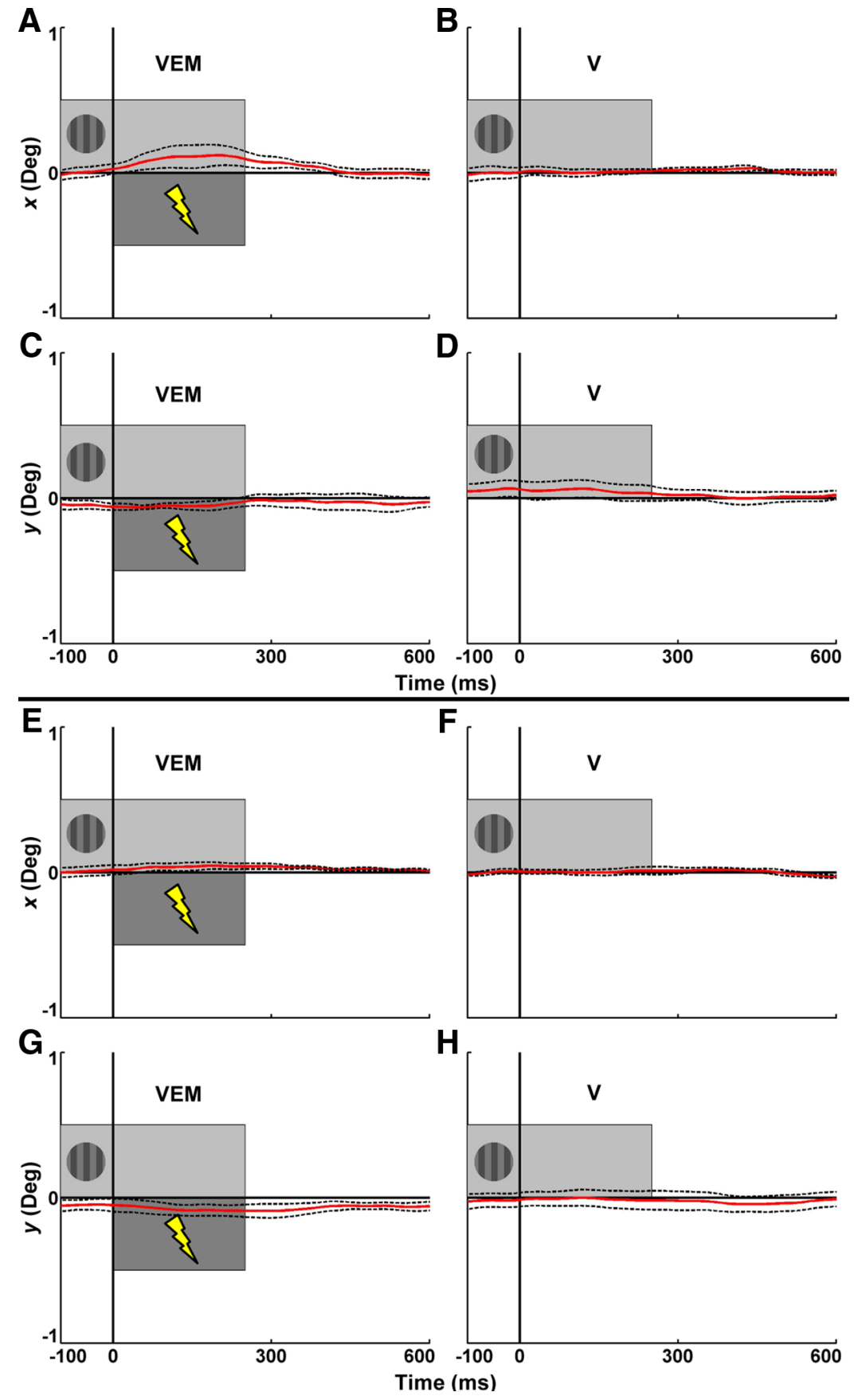

Figure 2. Average eye position aligned to EM onset. $\boldsymbol{A}, \boldsymbol{B}$, Mean horizontal eye trace for MM1; $\boldsymbol{C}, \boldsymbol{D}$, mean vertical eye trace for MM1. $\boldsymbol{E}, \boldsymbol{F}$, Mean horizontal eye trace for MM2; $\boldsymbol{G}, \boldsymbol{H}$, mean vertical eye trace for MM2. The solid red line plots mean eye position across all sessions and contrasts during VEM and V epochs; the dashed black lines show \pm SEM across the four electrodes stimulated. Traces have been aligned to EM onset (VEM) or the matching time in epochs without stimulation (V); total duration of FEF-EM is indicated by the bottom dark gray bar $(250 \mathrm{~ms})$. The top light gray bar indicates the period of visual stimulation (VEM, V); visual onset, at $-133 \mathrm{~ms}$ relative to $E M$ onset, is not shown. Any run showing a significant difference between the two conditions at any time point was removed ( $p>0.05$, corrected for multiple comparisons across time points; two-sample, two-tailed $t$ test across all acquired trials). These mean traces from the remaining runs show no significant difference at the aggregate level. Deg, Degree.

where $c$ is the fractional contrast level, $c_{50}$ is the contrast at which the response curve attains half of its maximum, $R_{\max }$ is the maximum response of the region, and $b$ is the baseline response value. When fitting the $\mathrm{V}$ conditions, we omitted the baseline response parameter because the $0 \% \mathrm{~V}$ condition was used as reference. This equation is a simplified form of the more general one used to fit contrast response data from single-cell recordings (Albrecht and Hamilton, 1982). As an estimate of 
Table 1. Fixation behavior during fMRI runs

\begin{tabular}{|c|c|c|c|c|c|c|c|c|c|c|}
\hline \multirow[b]{2}{*}{ Subject } & \multirow[b]{2}{*}{$n$} & \multicolumn{3}{|l|}{ \% Fixation } & \multicolumn{2}{|c|}{ Saccades/min } & \multicolumn{2}{|l|}{$\sigma_{x}\left({ }^{\circ}\right)$} & \multicolumn{2}{|l|}{$\sigma_{y}\left({ }^{\circ}\right)$} \\
\hline & & Conditions & Median & $p$ & Median & $p$ & Median & $p$ & Median & $p$ \\
\hline \multirow[t]{2}{*}{ MM1 } & 63 & VEM & 88.0 & 0.40 & 9.8 & 0.25 & 0.20 & 0.51 & 0.34 & 0.92 \\
\hline & & V & 86.2 & & 10.3 & & 0.20 & & 0.34 & \\
\hline \multirow[t]{2}{*}{ MM2 } & 78 & VEM & 87.1 & 0.53 & 10.9 & 0.90 & 0.25 & 0.81 & 0.39 & 0.69 \\
\hline & & V & 86.2 & & 11.0 & & 0.24 & & 0.40 & \\
\hline
\end{tabular}

$n$ is the number of independent fMRI runs collected from each subject. Percentage fixation was calculated using a $2^{\circ} \times 2^{\circ}$ window; $\sigma_{x}$ and $\sigma_{y}$ are the mean SD of eye position along the $x$ and $y$ eye axes, across all repetitions of a condition within a given run. A one-way ANOVA on each condition pair showed no significant differences $(p>0.05)$ in any of the distributions.

A

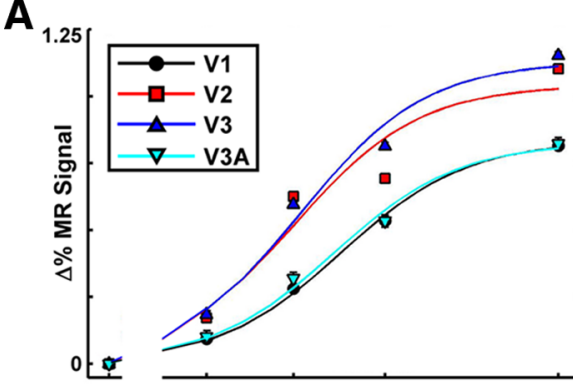

B
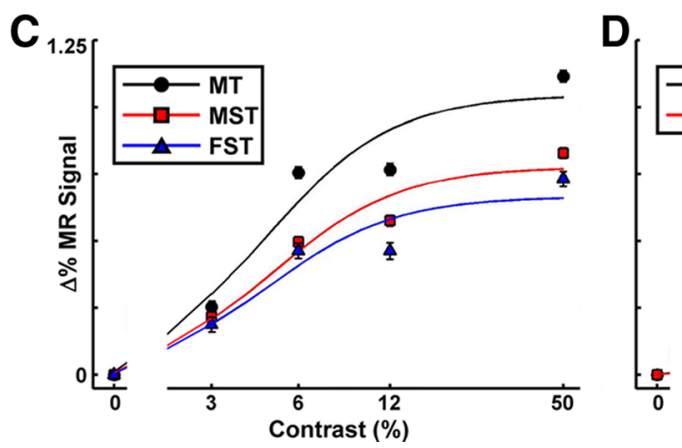

D.
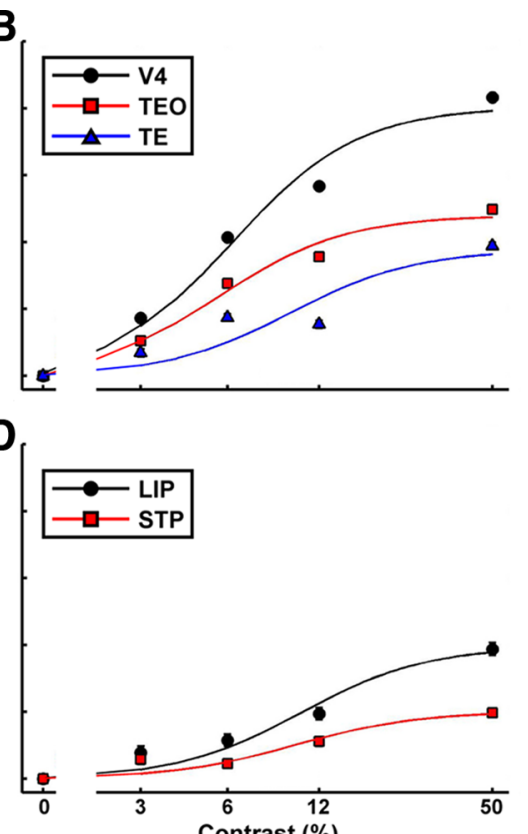

Figure 3. Contrast response functions in different visual areas. Percentage change in MR signal with respect to the baseline, or $0 \%$ contrast, condition as a function of luminance contrast for 12 visual cortical areas, including the following: early visual areas V1, V2, V3, and V3A ( $\boldsymbol{A})$; ventral extrastriate areas V4, TE0, and TE (B); areas within the STS, including MT, MST, and FST (C); and higher-order areas LIP and STP $(\boldsymbol{D})$. See Materials and Methods for a full description of how areal borders were determined. Each trace plots the mean response for all visual voxels localized in a given area, from both subjects across all sessions (only one stimulus location was used in each session). The smooth curves show the best-fitting Naka-Rushton function. Error bars indicate 1 SEM across epochs, and some error bars are smaller than the symbol used. Note that the $x$-axis has a logarithmic scale, with a break between 0 and $3 \%$ contrast.

how well Equation 1 described the fMRI response in each region, we calculated an adjusted $R^{2}$ value $\left(R_{\text {adj }}^{2}\right)$; this quantity describes how much of the variance in the data is captured by the model, with a penalty for the use of additional model parameters. To estimate $95 \%$ confidence intervals for all parameters, we implemented a nonparametric bootstrap algorithm (Efron and Tibshirani, 1993). In a particular region, the visual voxel population was randomly sampled with replacement, and the bestfitting parameters were calculated for that particular sample. We repeated this sampling 1000 times and determined the 2.5 and $97.5 \%$ quantile values from the resulting parameter distribution to indicate the confidence interval.

Receiver operator characteristic analysis. As an additional estimate of the difference between the distributions of V and VEM percentage change in MR signal values at a particular contrast level for the voxels in a given functional brain region, we calculated the area under a receiver operator characteristic (ROC) curve (McNicol, 1972). An ROC curve is determined by plotting the percentage of one distribution less than a given criterion value as a function of the percentage of the second distribution less than that same criterion, for all possible criterion values in the distributions. The area under the ROC curve then reflects how separate or distinguishable the two distributions are. An area of 0.5 means that the $\mathrm{V}$ and VEM distributions could not be discriminated at that contrast level in that region; deviations from 0.5 indicate increasing discriminability, with maximum possible values of 0 or 1 . To include the $0 \%$ contrast values in this analysis, we recalculated all our signal change values with respect to the mean fMRI signal value across all 10 stimulus conditions rather than the $0 \% \mathrm{~V}$ baseline condition before computing ROC curves. To estimate $95 \%$ confidence intervals on each of the ROC areas, we again used the nonparametric bootstrap algorithm $(n=$ 1000) (Efron and Tibshirani, 1993) described above.

\section{Results}

In both monkeys, we collected data associated with four stimulus locations (Fig. $1 B, C)$, corresponding to four implanted FEF electrodes and associated MFs, over six fMRI sessions. Stimuli were spread across the left visual field, contralateral to the side of the brain in which we implanted the electrodes, with examples in the upper and lower visual field and along the horizontal meridian. Eccentricities ranged from 1.8 to 15.0 visual degrees in MM1, and from 3.5 to 7.3 visual degrees in MM2. Saccade vectors were for the most part larger in MM1 than in MM2, suggesting that we stimulated slightly different parts of the FEF in the two subjects. Because of this small number of samples, we did not attempt to distinguish between eccentricities and polar angles and instead included all stimulus locations in one analysis (Fig. $1 B, C$ ).

For MM1, data from three identical sessions were combined for one target location, and data for the remaining three stimuli were each collected in a single session. For MM2, data for two stimulus locations were collected in individual sessions, and the remaining two locations were each collected over two sessions. On average, we collected 17.3 and 16.2 runs per session in MM1 and MM2, of which 10.7 and 12.9 runs per session were included in the analysis presented here. Inclusion criteria were based on a run-by-run analysis of the eye monitoring data recorded during each run. Runs in which the monkey's eye position at any time point in the stimulation sequence $(1 \mathrm{~s}$, sampled at $120 \mathrm{~Hz}$ ) was significantly different between $\mathrm{V}$ and VEM epochs were excluded from additional analysis (see Materials and Methods) (Fig. 2).

\section{Visual-only contrast response functions}

For each stimulus location, we identified a population of visual voxels for additional analysis from the fMRI contrast defined by the main visual effect at the highest luminance contrast (that is, $50 \% \mathrm{VEM}+50 \% \mathrm{~V}$ vs $0 \% \mathrm{VEM}+0 \% \mathrm{~V})$. Using this population, we extracted the percentage change in MR signal with respect to 
the fixation only or $0 \% \mathrm{~V}$ condition for each voxel at each of the other luminance contrast levels presented to the subjects. For each cortical area of interest, we then pooled the population of visual voxels across all sessions from both subjects and computed the mean response as a function of contrast to generate a V CRF.

Figure 3 shows the average CRF for each of the 12 cortical areas identified, grouped according to approximate cortical hierarchy (Felleman and Van Essen, 1991) and plotted on the same scale for comparative purposes. The smooth curves were calculated from the best fitting model to Equation 1 with the baseline parameter $b$ omitted (see Materials and Methods). Figure $3 \mathrm{~A}$ shows the response for early visual areas, including areas V1, V2, V3 and V3A. The CRFs for V2 and V3 were relatively similar to each other, as were the CRFs for V1 and V3A. Figure $3 B$ shows the response functions for visual areas in the ventral stream leading into inferotemporal (IT) cortex, including areas V4, temporaloccipital area (TEO), and temporal cortex (TE). The V4 CRF was qualitatively similar to the response seen for area V3. The two areas located more rostrally in IT cortex showed a decreased response to the contrast stimuli, with area TE showing the lowest contrast response. This low level of fMRI activity was likely because our gratings were non-optimal stimuli for driving these more object-sensitive areas (Vogels and Orban, 1994).

Figure $3 C$ shows the response functions for visual areas in the superior temporal sulcus (STS) more commonly identified with the dorsal stream, such as the middle temporal area (MT) and medial superior temporal area (MST), as well as the fundus of superior temporal sulcus (FST). Area MT showed the highest contrast sensitivity and the largest overall fMRI activation of all the areas sampled (mean response of $0.578 \pm 0.011 \%$ change in MR signal across all contrasts). MST and FST had lower contrast responses, with area FST being the least responsive of this group, although qualitatively quite similar to area MT in terms of change in response between contrasts. Figure $3 D$ shows the response functions for the two highest-order visual areas sampled, the lateral intraparietal area (LIP) in the lateral bank in the intraparietal sulcus and superior temporal polysensory area (STP) in the upper bank of the STS. These two areas were the least responsive of all to luminance contrast, perhaps reflecting their role in integrating information from multiple sensory modalities (mostly STP) or their large receptive field (RF) sizes compared with the small stimuli size we used.

Despite considerable differences in methodology and the stimuli used, we attempted to compare the CRFs obtained here with CRFs collected in previous human (Tootell et al., 1995; Buracas and Boynton, 2007; Li et al., 2008) and nonhuman primate studies (Albrecht and Hamilton, 1982; Sclar et al., 1990; Williford and Maunsell, 2006; Palmer et al., 2007) (supplemental Fig. S1 and discussion in the accompanying supplemental data, available at www.jneurosci.org as supplemental material). In general, we found good agreement with past human fMRI studies in early visual areas for which human fMRI data are available. In area MT, our monkey fMRI data matched more closely the single-unit results than the human fMRI data.

Table 2 gives a summary of the best-fitting parameters for each area from the Naka-Rushton model, with 95\% confidence intervals estimated from a nonparametric bootstrap algorithm $(n=1000)$. Several of the qualitative trends described above are apparent in these modeling results. Area MT had the sharpest rising CRF and, accordingly, the lowest $c_{50}$ value, indicating that this area is the most contrast sensitive of all visual areas measured. Extrastriate areas V2, V3, and V4 all had similar CRFs and thus relatively similar model parameters. Some of the higher-order
Table 2. Naka-Rushton model parameters from the best fit to the percentage change in MR signal values in the $\mathbf{V}$ conditions for each visual area

\begin{tabular}{lllllll}
\hline & \multicolumn{2}{l}{ (\% MR signal) } & & \multicolumn{3}{l}{ (\% Contrast) } \\
\cline { 2 - 3 } Visual area & $R_{\max }$ & $95 \% \mathrm{Cl}$ & & $c_{50}$ & $95 \% \mathrm{Cl}$ & $R_{\text {adj }}{ }^{2}$ \\
\hline V1 & 0.83 & $(0.81,0.86)$ & & 8.8 & $(8.6,9.1)$ & 0.99 \\
V2 & 1.04 & $(1.00,1.08)$ & & 6.1 & $(5.8,6.3)$ & 0.84 \\
V3 & 1.13 & $(1.08,1.17)$ & & 6.4 & $(6.1,6.7)$ & 0.95 \\
V3A & 0.83 & $(0.75,0.91)$ & & 8.3 & $(7.6,9.2)$ & 0.98 \\
V4 & 1.01 & $(0.97,1.04)$ & & 6.3 & $(6.0,6.6)$ & 0.94 \\
MT & 1.05 & $(0.99,1.10)$ & & 4.7 & $(4.5,5.0)$ & 0.84 \\
LIP & 0.49 & $(0.46,0.53)$ & & 10.8 & $(9.1,12.7)$ & 0.86 \\
MST & 0.78 & $(0.73,0.83)$ & & 5.0 & $(4.6,5.4)$ & 0.90 \\
FST & 0.67 & $(0.62,0.71)$ & & 4.8 & $(4.1,5.6)$ & 0.79 \\
TE0 & 0.60 & $(0.57,0.63)$ & & 5.7 & $(5.2,6.2)$ & 0.95 \\
TE & 0.47 & $(0.44,0.51)$ & & 9.9 & $(7.6,12.5)$ & 0.53 \\
STP & 0.25 & $(0.22,0.28)$ & & 10.3 & $(7.3,14.1)$ & 0.75 \\
\hline
\end{tabular}

Best fit was determined by minimizing the sum of the squared error. Confidence intervals ( $\mathrm{Cl}$ ) were estimated using a nonparametric bootstrap algorithm ( $n=1000$; see Materials and Methods).

areas that showed the least responsivity to contrast (LIP, TE, and STP in particular) had the highest $c_{50}$ and lowest $R_{\max }$ values.

\section{Contrast response functions with FEF-EM: area V1}

From the same population of visual voxels, we also extracted the percentage change in MR signal for the epochs that combined a visual stimulus with simultaneous electrical stimulation of the FEF location with a matching movement field. We again calculated a mean response at each contrast level, across all stimulus locations in both subjects, to generate a VEM CRF. Figure $4 \mathrm{~A}$ shows for area V1 the resulting VEM CRF, along with the V CRF from Figure $3 A$ for comparison. Note that in this and following figure, we plot only the measured mean VEM and V responses and their derivatives, rather than any fitted functions, to give the most accurate indication of how FEF-EM modulated the cortical contrast response. For area V1, the FEF-EM-dependent modulation of visually driven activity varied as a function of the underlying luminance contrast of the visual stimulus. For the low-contrast stimuli, we measured an increase in mean fMRI activation (from $0.094 \pm 0.010$ to $0.221 \pm 0.011 \%$ change in MR signal at $3 \%$ luminance contrast, without and with FEF-EM, respectively), whereas for the highest contrast stimuli, we measured a decrease in mean fMRI activation (from $0.816 \pm 0.011$ to $0.695 \pm 0.010 \%$ change in MR signal at $50 \%$ luminance contrast). The crossover point, when activation in the visual-only condition would presumably exceed activation in the combined visual and FEF-EM condition, was somewhere between 6 and $12 \%$ luminance contrast.

Next, we computed the difference between these two CRFs, shown by the black trace in Figure $4 B$, and used a two-way ANOVA to assess whether there was a significant interaction between FEF-EM and visual stimulation, relative to the $0 \% \mathrm{~V}$ and $0 \%$ VEM conditions $(p<0.05$; factors EM and visual stimulation). A positive interaction, here indicating a larger response in the combined VEM condition at a given contrast than the sum of the responses in the $\mathrm{V}$ condition at that same contrast and the $0 \%$ VEM condition, is noted by the presence of a black filled square at each contrast level. A negative interaction, here indicating the opposite effect, is noted by an open black square. In area V1, we found that there was a significant interaction at the $3 \%$ (positive), $12 \%$ (negative), and 50\% (negative) luminance contrast levels. In agreement with our previous work (Ekstrom et al., 2008), no significant difference was found in V1 at the $0 \%$ contrast level, which indicates no EM-only effect or so-called baseline shift in this region ( $p>0.05$, one-sample, two-tailed $t$ test). 


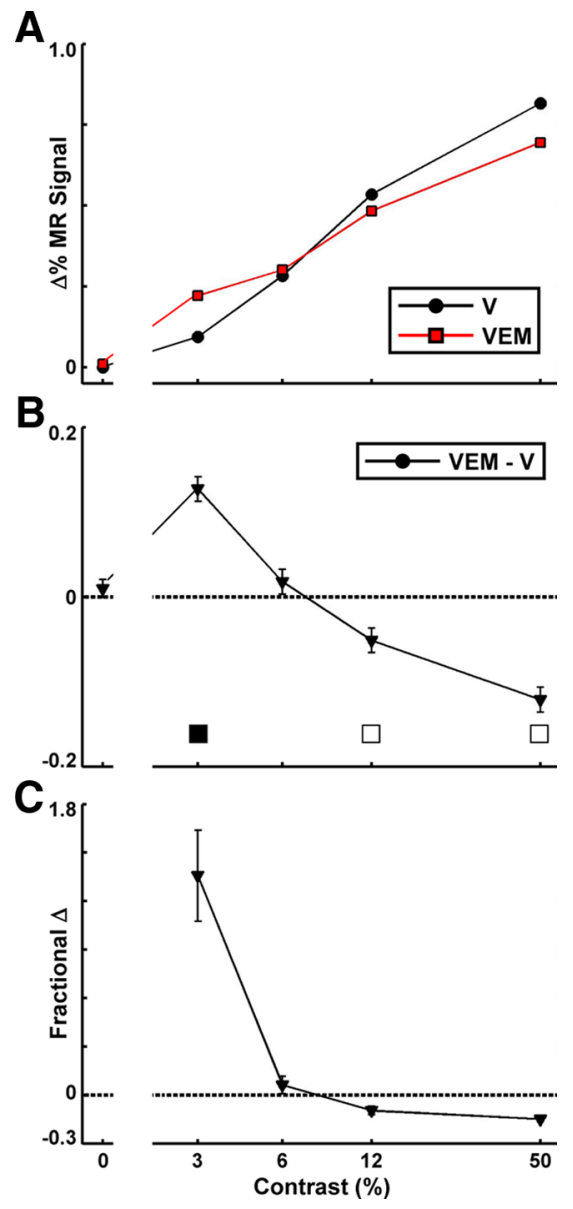

Figure 4. Modulation of the contrast response function in area $\mathrm{V} 1$ by microstimulation of the FEF. $A$, Percentage change in MR signal with respect to the baseline condition in the visual-only (V; black curve) and visual with FEF-EM (VEM; red curve) conditions as a function of luminance contrast. Each trace plots the mean response for all visual voxels localized in V1, from both subjects across all sessions. The fMRI data used to derive the area V4 curves were also used to derive the $\mathrm{V} 4$ curves presented by Ekstrom et al. (2008), their Figure $5 B$. The same holds true for the V4 data presented in Figure 5A. $\boldsymbol{B}$, Difference in fMRI activity between the VEM and V conditions at each contrast level (a subtraction of the two curves in $\boldsymbol{A}$ ). Filled/open squares below the traces indicate a significant positive/negative interaction between the effects of $\mathrm{EM}$ and $V$ on the activity (black symbols) ( $p<0.05$, two-way ANOVA). C, Fractional change in percentage MR signal, (VEM - V) $/ V$, indicating the effective change in fMRI activity caused by FEF-EM relative to the visual-only activation level. In all panels, error bars indicate 1 SEM across epochs; some error bars are smaller than the symbol used.

After comparing the difference between the VEM and V epochs, we also computed the fractional change this difference represents in terms of the underlying visual-only activation. For a particular area, we reasoned that a large change to a small initial activation would convey more information to any region reading out these signals than a change of equal magnitude on top of an already large activation. Figure $4 C$ shows this fractional change as a function of contrast for area $\mathrm{V} 1$. The use of the $0 \% \mathrm{~V}$ condition as our reference point precluded us from calculating this value at $0 \%$ contrast (although, as shown in Fig. $4 B$, the difference between $\mathrm{V}$ and VEM at this contrast is clearly quite small) but, for all other contrast levels, we calculated a fractional change response function. Using this measure, we found that the modulations attributable to FEF-EM at the lowest contrast became even more apparent: the change in activation caused by FEF-EM in V1 represented a $1.4 \pm 0.3$-fold increase in fMRI activity. Conversely, the response at high contrast decreased by $0.15 \pm 0.02$ times the initial visual-only activation, which is a much smaller effect than that observed at low contrast.

In addition to modulations of the mean response, another way to characterize how well an ideal observer could read out changes attributable to FEF-EM is to determine how separate or distinguishable the distributions of V and VEM signal change values are for the population of visual voxels. We estimated this distinguishability by implementing an ROC analysis (see Materials and Methods). Supplemental Figure S2 (available at www.jneurosci. org as supplemental material) shows a series of ROC curves for each contrast level, and supplemental Figure S3A (black trace) (available at www.jneurosci.org as supplemental material) shows the resulting area under each ROC curve as a function of luminance contrast for area V1. In general, we observed in area V1 a very similar trend with this measure as with the arithmetic difference measure. The VEM and V signal change distributions were most distinguishable for the lowest contrast stimuli, with VEM greater than $\mathrm{V}$, and for the highest contrast stimuli, with $\mathrm{V}$ greater than VEM. The largest suppressive effect of FEF-EM at high contrast (i.e., $\mathrm{V}$ greater than VEM) was observed in area V1.

\section{Contrast response functions with FEF-EM: all visual areas}

We extended the above analysis to all visual cortical areas that were driven by the $50 \%$ contrast gratings. Figure 5 shows difference CRF and fractional change plots for the 12 cortical areas first identified in Figure 3, with the corresponding plots for V1 repeated from Figure 4 for comparative purposes. We have grouped areas together that are close to each other in terms of cortical hierarchy and that show similar qualitative features in their difference CRFs. In all areas except STP, we observed the largest increase in fMRI activity in the VEM epochs at the $3 \%$ luminance contrast level, whereas the highest-contrast stimuli produced no or even negative interactions, which indicate a reduction in fMRI activity attributable to EM. We think that these high-contrast observations are not likely attributable to a ceiling effect of our fMRI measurements, given that we found a decrease in activation in some regions and were able to measure much larger percentage signal change values in some areas in our previous study (Ekstrom et al., 2008, their Fig. 1C). In several areas, including V3, V4, MT, MST, FST, LIP, and STP, we measured a significant baseline shift attributable to FEF-EM $(p<0.05$, onesample, two-tailed $t$ test). This group of areas is monosynaptically connected with the FEF (Huerta et al., 1987; Schall et al., 1995; Stanton et al., 1995), and so a robust, fMRI-detectable, EM-only effect is expected here (Tolias et al., 2005; Ekstrom et al., 2008; Moeller et al., 2008). In four areas, including V2, MT, MST, and FST, we observed a significant negative interaction at the $6 \%$ contrast level that does not seem to match the trend established by the next lower and higher contrasts. Although we have no obvious explanation for this effect observed in both monkeys, we note that this feature is common to mainly dorsal stream areas that are strongly interconnected anatomically (Ungerleider and Desimone, 1986a,b; Boussaoud et al., 1990).

As we did for the $\mathrm{V}$ conditions, we fit the Naka-Rushton model (Eq. 1) to the mean VEM response in each area (supplemental Table S1, available at www.jneurosci.org as supplemental material). In this instance, we included the baseline parameter $b$ in the model fit. In general, Equation 1 provided a less satisfactory description of the data in the VEM conditions than in the $\mathrm{V}$ conditions. This deficiency is indicated by the general decrease in $R_{\mathrm{adj}^{2}}$ values for the VEM model fits compared with the $\mathrm{V}$ fits. Figure $4 A$ illustrates well the features of the data responsible for this difference in fit quality. The Naka-Rushton model (Naka and 
Rushton, 1966) and more general hyperbolic ratio models (Albrecht and Hamilton, 1982) produce a smooth sigmoid function that seems to characterize well the visual-only CRF in cortical visual areas. With the addition of FEF-EM, however, the model does not well capture both the pronounced rise in activity that we observed at $3 \%$ contrast coupled with the decrease in activity at high contrast. As a result, the best-fitting baseline parameter $b$ in V1 (supplemental Table S1, available at www.jneurosci.org as supplemental material) is found to be non-zero, which disagrees with our earlier statistical analysis and our previous findings (Ekstrom et al., 2008). Furthermore, many of the $c_{50}$ values (supplemental Table S1, available at www.jneurosci.org as supplemental material) do not reflect the downward shift expected by the increase in activity observed at 3\% contrast. Thus, although Equation 1 appears to adequately model the $\mathrm{V}$ data, this does not hold not true for the VEM data in all visual areas.

As before, we computed the fractional change response functions for all 12 areas, by dividing the difference between VEM and $\mathrm{V}$ epochs by the underlying visualonly activation. These response functions are presented in Figure $5 D-F$, following the same grouping of areas used above. The response function for $\mathrm{V} 1$ is repeated from Figure $4 C$ for comparative purposes. These curves further accentuate the pattern seen in the preceding graphs. For most areas, the largest enhancement of fMRI activity by FEF-EM above the underlying visual response was seen for the lowest contrast stimuli used. In supplemental Figure S4 (available at www.jneurosci.org as supplemental material), we show this same analysis for MM1 and MM2 individually. Despite the relatively different distribution of saccade amplitudes between the subjects, we observed approximately the same modulation pattern in both. At the highest contrast, however, we do see more examples of suppression of fMRI activity in MM2 than in MM1.

Finally, for each area, we computed an ROC curve using the VEM and $V$ signal change distributions at each contrast level and associated areas under the ROC curve (supplemental Fig. S3, available at www.jneurosci.org as supplemental material). This analysis produced essentially the same results as the arithmetic difference measure outlined above.

\section{Generalized CRF for visual cortex}

By pooling all voxels from the identified cortical areas, we calculated an overall CRF, difference CRF, and fractional change function for all visually driven voxels in occipital cortex. The overall CRF (Fig. 6A, black circles) gives an accurate estimate of the overall response of the visually driven areas in cortex, although it is biased toward the larger visual areas. The black trace in Figure $6 \mathrm{~B}$ shows the overall difference CRF; black squares indicate the contrast levels with a significant interaction between EM and the visual response $(p<0.05$, two-way ANOVA). We observed a

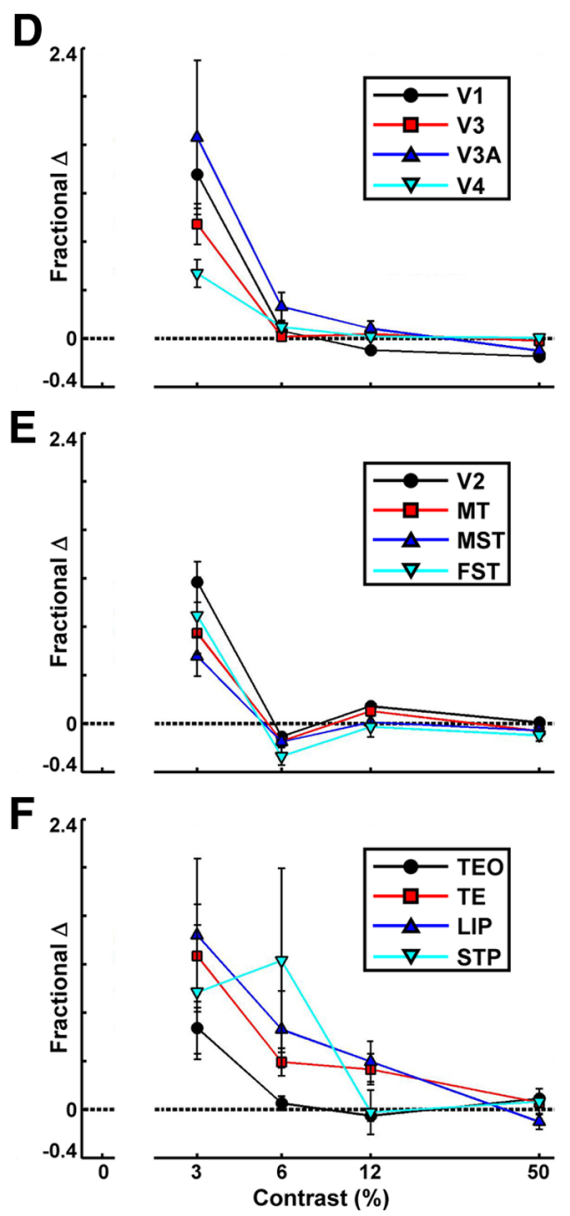

Contrast (\%)

D

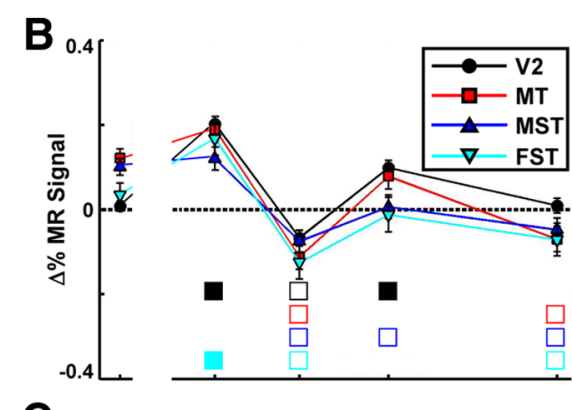

Contrast $(\%)$

Figure 5. Modulation of contrast response functions in multiple visual areas by microstimulation of the FEF. $A-C$, The difference in fMRI activity between the VEM and V conditions at each contrast level (Fig. $4 B$; area V1 is repeated here for comparative purposes) for the 12 areas defined in Figure 3. Filled/open squares below the traces indicate a significant positive/negative interaction between factors EM and $V$ relative to the $0 \%$ values ( $p<0.05$; two-way ANOVA). $\mathbf{D}-\boldsymbol{F}$, Fractional change in percentage MR signal $[(\mathrm{VEM}-\mathrm{V}) / \mathrm{V}]$. In all panels, error bars indicate 1 SEM across epochs.

significant positive interaction at $3 \%$ luminance contrast and a significant negative interaction at $50 \%$ luminance contrast. In addition, we measured a significant overall baseline shift at $0 \%$ contrast ( $p<0.05$, one-sample, two-tailed $t$ test). Figure $6 C$ shows the corresponding fractional change response function.

In an additional analysis, we split our population of visual voxels into highly and weakly visually responsive groups, on the basis of the $t$ score of each voxel in the localizer contrast (that is, $50 \% \mathrm{VEM}+50 \% \mathrm{~V}$ vs $0 \% \mathrm{VEM}+0 \% \mathrm{~V}$ ). All voxels that exceeded a $p$ value of 0.05 at the corrected level were included in the high population (in other words, visually very well driven voxels). The remaining visual voxels (that is, 0.05 , corrected $<p<0.05$, uncorrected) were included in the low population. This split resulted in an approximately equal-sized pool of voxels in each population. In Figure $6 \mathrm{~A}$, we also present the CRFs for these two subdivisions of our larger population along with the best-fitting model for each group (red and blue curves). Figure $6 B$ also shows the difference CRFs for these additional populations, and Figure $6 C$ shows the fractional change functions for each group. Table 3 gives the best-fitting model parameters to the $\mathrm{V}$ conditions, and supplemental Table S2 (available at www.jneurosci.org as supplemental material) shows the fit parameters for the VEM responses. Both the weakly and strongly driven voxels exhibited a baseline shift, or an increase in activity attributable to FEF-EM in the 


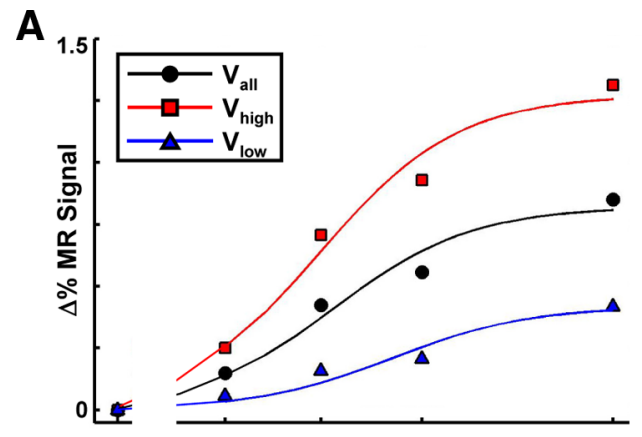

B
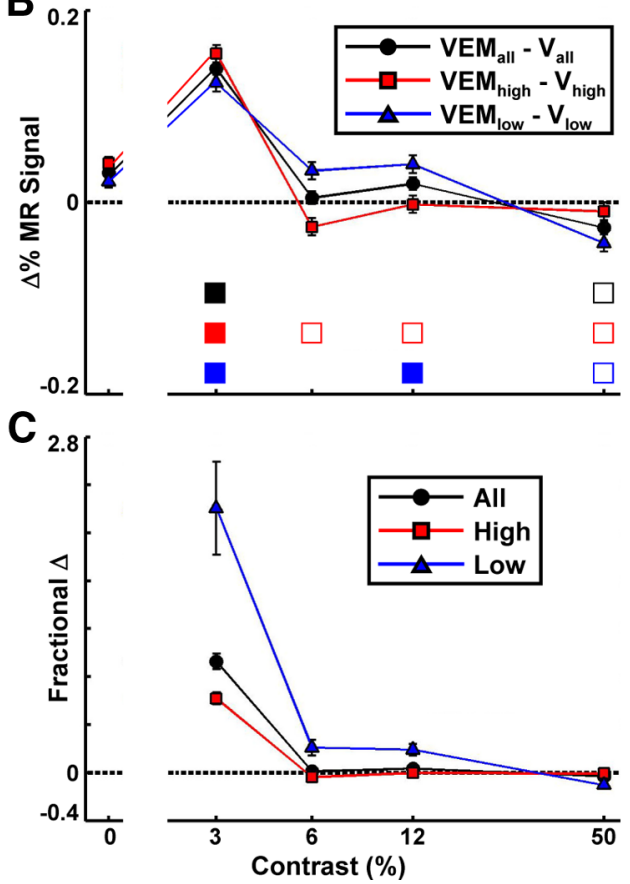

Figure 6. Contrast response functions for weakly and strongly visually driven voxels and their modulation by FEF microstimulation. $A$, Mean percentage change in MR signal for the $V$ conditions as a function of contrast, across both subjects and all visual voxels in the 12 visual areas defined in Figure 4 . Areas with more active voxels are weighted more. $V_{\text {all }}$ represents all visual voxels ( $p<0.05$, uncorrected). $V_{\text {high }}$ represents the best visually driven voxels ( $p<$ 0.05 , corrected). $V_{\text {low }}$ represents all remaining voxels from the visual voxels pool not included in $V_{\text {high }}(0.05$, corrected $<p<0.05$, uncorrected); on the cortical surface, these voxels are primarily located in annuli surrounding the best-driven population. The smooth curves show the best-fitting Naka-Rushton functions. $\boldsymbol{B}$, Mean difference between VEM and V conditions. Filled/open squares below the traces indicate a significant positive/negative interaction between factors EM and V ( $p<0.05$, two-way ANOVA). C, Fractional change in percentage MR signal. In all panels, error bars indicate 1 SEM across epochs.

Table 3. Naka-Rushton model parameters from the best fit to percentage change in MR signal values in V conditions for the mean response in the all, high, and low populations of voxels

\begin{tabular}{lllllll}
\hline & \multicolumn{2}{l}{ (\% MR signal) } & & \multicolumn{2}{l}{ (\% Contrast) } \\
\cline { 2 - 3 } Voxel group & $R_{\max }$ & $95 \% \mathrm{Cl}$ & & $c_{50}$ & $95 \% \mathrm{Cl}$ & $R_{\text {adj }}^{2}$ \\
\hline All & 0.82 & $(0.81,0.83)$ & & 6.6 & $(6.5,6.7)$ & 0.92 \\
High & 1.27 & $(1.25,1.29)$ & & 6.0 & $(5.9,6.1)$ & 0.95 \\
Low & 0.42 & $(0.41,0.43)$ & & 10.1 & $(9.4,10.8)$ & 0.85
\end{tabular}

Best fit was determined by minimizing the sum of the squared error. Confidence intervals $(\mathrm{Cl})$ were estimated using a nonparametric bootstrap algorithm ( $n=1000$; see Materials and Methods).

absence of visual stimulation ( $p<0.05$, one-sample, two-tailed $t$ test). Furthermore, we observed a remarkable difference in the effects of FEF-EM on the CRF of the low and high population of voxels. The low population displayed a significant positive inter-

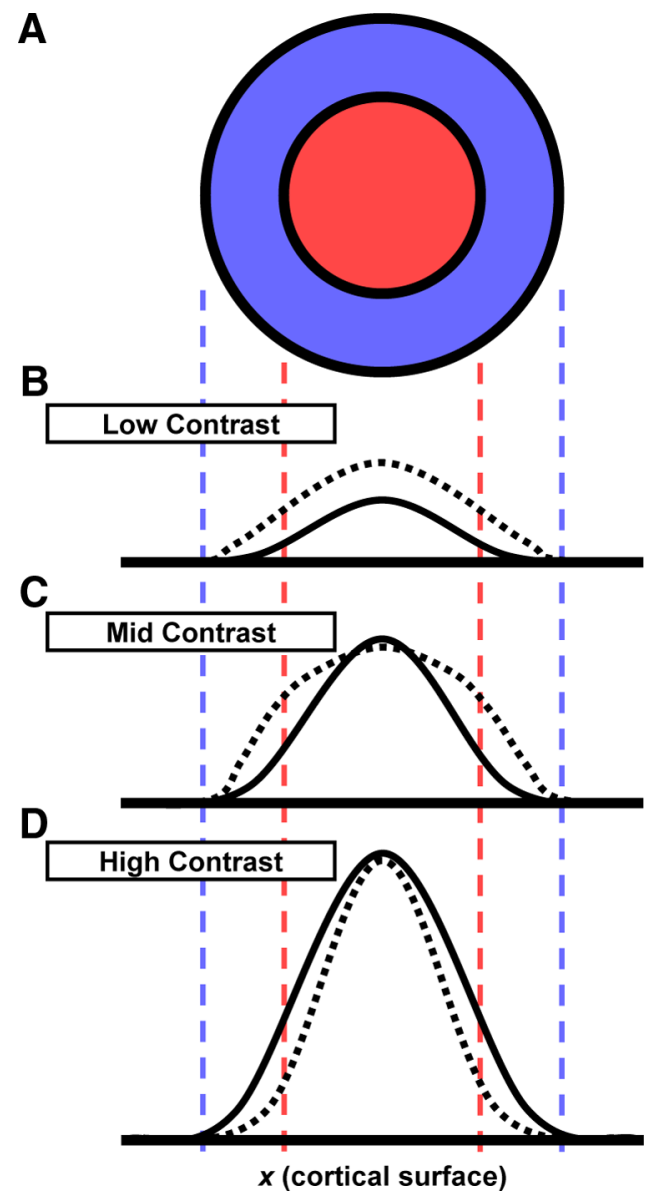

Figure 7. Schematic summary of the effect of FEF-EM. $A$, The concentric circles correspond to regions on the two-dimensional cortical surface representation of a visual stimulus (compare with Fig. 1D). Red/blue match the well/less well driven populations of visual voxels. $\boldsymbol{B}-\boldsymbol{D}$, Projecting this surface to one dimension yields three different activity profiles, depending on the stimulus contrast used (solid curve represents visual-only activation, and dotted curve the combined response to visual stimulation and FEF-EM).

action at 3 and $12 \%$ and a significant negative interaction at $50 \%$ $(p<0.05$, two-way ANOVA). For the high population, we also found a positive interaction at 3\%; however, we found a negative interaction at all three higher contrast levels $(p<0.05$, two-way ANOVA).

The results of the split-population analysis are summarized schematically in Figure 7. The colored circles represent the two populations of voxels on the cortical surface, with the highly responsive voxels in the center and the weakly responsive voxels in the margin of the schematic. The bottom panels of Figure 7 show a projection of the activity profile onto a single spatial dimension. For the lowest contrast stimuli, FEF-EM amplified the activity of both weakly and strongly driven voxels (Fig. 7B, compare solid, V-response curve with dotted, VEM-response curve). For the highest contrast stimuli (Fig. 7D), the macroscopic circuit switched to a discriminatory mode; the activity of all voxels was slightly suppressed, although weakly driven voxels were suppressed more. For intermediate contrasts (Fig. 7C), the results show a mixture of effects: the activity of weakly responsive voxels was still amplified, whereas strongly responsive voxels were slightly suppressed.

\section{Discussion}

Using CBV-weighted fMRI, we have measured response functions to visual stimuli at five luminance contrast levels in 12 de- 
fined functional areas of the macaque visual cortex. Furthermore, we observed how this contrast response was modulated by the simultaneous stimulation of FEF sites with movement fields at the location of the visual stimuli. We observed a significant baseline shift (i.e., at $0 \%$ contrast) attributable to FEF-EM in a subset of areas known to be well connected to the FEF, and in general we found the largest increases of fMRI activity for the lowest contrast stimuli that produce smallest amounts of visual drive in cortical areas. Activity for high-contrast stimuli, which produce the largest drive, were unaffected or reduced by FEF-EM.

\section{Methodological comparisons with previous studies}

With respect to commonly used attention models, such as contrast and response gain (Reynolds et al., 2000; Williford and Maunsell, 2006; Li et al., 2008), we note several key distinctions between such attention studies and our own. Our subjects performed a passive fixation task as we stimulated the FEF; hence, attention was not modulated here. Although FEF stimulation has been shown to produce results that physiologically resemble spatial attention effects in visual cortex (Moore et al., 2003; Armstrong et al., 2006; Armstrong and Moore, 2007), exact correspondence with an attention task has not been established (that is, the behavioral and physiological results after FEF-EM were obtained in separate experiments in these studies). Second, the comparison most often made during attention tasks is to direct attention toward and away from (typically to the contralateral visual field) the stimulus of interest. This approach holds the task difficulty constant and permits a comparison between neuronal responses to attended and ignored stimuli (Martínez-Trujillo and Treue, 2002). Here, we compared visual responses during the presence and absence of FEF stimulation, which would more closely parallel an attend-toward/no-attend design. Thus, our baseline condition at each contrast level was a visual-only response and presumably lacks the suppressive interactions between simultaneously presented stimuli (Luck et al., 1997; Kastner et al., 1998) as well as putative top-down inhibitory effects present during an attend-away condition.

Previous combined fMRI microstimulation experiments in monkeys (Tolias et al., 2005) revealed increased activity only in relatively proximal areas monosynaptically connected with the site of stimulation. In this and our previous study (Ekstrom et al., 2008), we observed FEF-EM-dependent modulations of visually driven activity in areas distant from the site of stimulation and connected with FEF through multiple synapses. Importantly, however, we only observed EM-only activity [i.e., in the absence of visual stimuli, similar to a previous study (Tolias et al., 2005)] in areas directly connected with FEF. Moreover, there are several methodological differences between our studies and that of Tolias and colleagues, including the use of awake versus anesthetized subjects and the use of relatively small versus large currents. Although speculative, an important factor may be that the microstimulation of presumptive feedback pathways allows the investigation of multisynaptic effects more easily than that of feedforward pathways, because the former may be more modulatory in nature and easier to capture with fMRI (Logothetis, 2008).

\section{Modulation of CRFs by FEF-EM}

The models that have been proposed to describe the effects of attention on neural activity in the visual cortex (Williford and Maunsell, 2006) provide a useful framework for describing the effects of FEF stimulation. As mentioned above, one model that has been proposed to explain the effects of attention on CRFs is the response gain model, which suggests that attention produces a proportional increase in response at all contrasts so that the effect is most pronounced at the highest contrasts. Under the conditions of our study, we did not observe evidence for a response gain model; instead, in many areas and in the overall response across visual cortex, we saw a reduction in activity at high contrast. An alternative is the contrast gain model, which proposes that attention produces an increase in the apparent contrast of a stimulus, causing a leftward shift of the entire CRF (Reynolds et al., 2000). The principal characteristic of a contrast gain effect is an increase in activity for stimuli at intermediate contrast, with little effect at baseline and saturation activity levels. We do observe common features with this model, specifically at lower and intermediate contrasts. The correspondence is not perfect, however, particularly at high contrast. As we noted above, many of the previous studies that investigated the effects of attention on CRFs compared attend-toward and attend-away conditions in the presence of multiple stimuli rather than an analog of the stimulation/no-stimulation design with a single stimulus used here. However, we cannot exclude the alternative explanation that FEF-EM does not reproduce all the effects of selective attention on neuronal activity in the visual cortex.

Recently, two models were proposed in an attempt to unify the apparently contradictory contrast gain and activity or response gain modulation of single-unit responses using divisive normalization of stimulus-driven activity (Lee and Maunsell, 2009; Reynolds and Heeger, 2009). In the Reynolds-Heeger model, excitatory and suppressive stimulus drive and the spatial spread of the attentional feedback all contribute to the observed response. For example, if an attended stimulus matches the RF size in a given area yet the attentional field is smaller than the stimulus, the model predicts a strong response gain. If, however, the stimulus is small relative to both the RF and attentional field, the model predicts a contrast gain effect. If one assumes that FEF-EM mimics spatial attention processes, the ReynoldsHeeger model would predict very different effects in different visual areas, depending on respective RF sizes relative to our $3^{\circ}$ radius stimuli. We observed, however, very similar FEF-EM effects in all areas, regardless of neuronal RF size (supplemental Fig. S5, available at www.jneurosci.org as supplemental material). Importantly, the size of the presumptive attentional field is a variable in the Reynolds-Heeger model that is difficult to assess in our experiments. Although this size was fixed for a given electrode, it may be larger than the largest RFs in any of the areas investigated (e.g., FST, MST, and STP). Future experiments in which both stimulus size and attentional field size are manipulated (e.g., by using variable currents or stimulating multiple electrodes simultaneously) would allow a more direct test of the Reynolds-Heeger model.

Our results, and specifically the schematic of Figure 7, bear resemblance to previous frameworks for center-surround interactions in rat somatosensory cortex (Simons, 1985; ArmstrongJames and Fox, 1987; Ghazanfar and Nicolelis, 1997; Moore et al., 1999), models for contrast-dependent interactions in area V1 (Somers et al., 1998; Schwabe et al., 2006), and the above Reynolds-Heeger model (Reynolds and Heeger, 2009). We suggest that the presumptive feedback connections activated from area FEF (1) amplify all the input at low levels of visual drive, (2) sharpen salient responses at high levels of activity (i.e., increase discriminability), and (3) cause a mixture of these effects at intermediate activity levels. Such a mixture is consistent with our previous findings (Ekstrom et al., 2008), in which we used chromatic stimuli ( $\sim 31 \%$ luminance contrast) and observed a slight 
suppression of the fMRI response at the center of the representation of these stimuli and an enhancement of activity in the surrounding less well activated voxels. These findings may appear to be at odds with a previous report of FEF-EM, causing the largest enhancement of V4 neuronal firing rates for optimally oriented stimuli (Moore and Armstrong, 2003). We note, however, that the use of fMRI precludes the stimulation of all neurons within a voxel with optimal stimulus orientation, so that the apparent discrepancy may be attributable to the combination of neuronal responses with a range of stimulus preferences.

Several predictions relating to the modulation of a stimulus representation and perception attributable to increased FEF activity, either through EM in monkeys or transcranial magnetic stimulation in humans (Grosbras and Paus, 2002; Ruff et al., 2006; Silvanto et al., 2006; Taylor et al., 2007), are possible from this operating schematic (Fig. 7). First, low-contrast stimuli in isolation should be perceived more readily, similar to the facilitated detection of luminance changes after FEF-EM (Moore and Fallah, 2004) and as seen in many attention experiments (Bashinski and Bacharach, 1980; Hawkins et al., 1990; Müller and Humphreys, 1991; Handy et al., 1996). A second prediction arises from the comparison of the mid- and high-contrast regimens. Specifically, a discrimination task using stimuli comprising of a central region and annulus, with the discrimination made in the annular compartment, should be differentially impaired or facilitated by FEF output depending on the contrast used. Parallels of this prediction can be seen in recent work combining fMRI and TMS of human FEF (Ruff et al., 2006).

Our results thus strengthen theories proposing that increased output from areas responsible for oculomotor control modulates incoming visual activity (Rizzolatti et al., 1987; Corbetta et al., 1998; Moore and Armstrong, 2003; Cavanaugh and Wurtz, 2004; Ruff et al., 2006). Moreover, this top-down modulation depends on the underlying sensory drive so that neuronal activity in visual cortex is amplified at low-stimulus contrast but sharpened at high-contrast levels.

\section{References}

Albrecht DG, Hamilton DB (1982) Striate cortex of monkey and cat: contrast response function. J Neurophysiol 48:217-237.

Armstrong KM, Moore T (2007) Rapid enhancement of visual cortical response discriminability by microstimulation of the frontal eye field. Proc Natl Acad Sci U S A 104:9499-9504.

Armstrong KM, Fitzgerald JK, Moore T (2006) Changes in visual receptive fields with microstimulation of frontal cortex. Neuron 50:791-798.

Armstrong-James M, Fox K (1987) Spatiotemporal convergence and divergence in the rat S1 "barrel" cortex. J Comp Neurol 263:265-281.

Bartlett JR, DeYoe EA, Doty RW, Lee BB, Lewine JD, Negrão N, Overman WH Jr (2005) Psychophysics of electrical stimulation of striate cortex in macaques. J Neurophysiol 94:3430-3442.

Bashinski HS, Bacharach VR (1980) Enhancement of perceptual sensitivity as the result of selectively attending to spatial locations. Percept Psychophys 28:241-248.

Boussaoud D, Ungerleider LG, Desimone R (1990) Pathways for motion analysis: cortical connections of the medial superior temporal and fundus of the superior temporal visual areas in the macaque. J Comp Neurol 296:462-495.

Bruce CJ, Goldberg ME, Bushnell MC, Stanton GB (1985) Primate frontal eye fields. II. Physiological and anatomical correlates of electrically evoked eye movements. J Neurophysiol 54:714-734.

Buracas GT, Boynton GM (2007) The effect of spatial attention on contrast response functions in human visual cortex. J Neurosci 27:93-97.

Cavanaugh J, Wurtz RH (2004) Subcortical modulation of attention counters change blindness. J Neurosci 24:11236-11243.

Chef d'Hotel C, Hermosillo G, Faugeras O (2002) Flows of diffeomorphisms for multimodal image registration. Proc IEEE Int Symp Biomed Imaging 7 8:21-26.
Corbetta M, Shulman GL (2002) Control of goal-directed and stimulusdriven attention in the brain. Nat Rev Neurosci 3:201-215.

Corbetta M, Akbudak E, Conturo TE, Snyder AZ, Ollinger JM, Drury HA, Linenweber MR, Petersen SE, Raichle ME, Van Essen DC, Shulman GL (1998) A common network of functional areas for attention and eye movements. Neuron 21:761-773.

Dale AM, Fischl B, Sereno MI (1999) Cortical surface-based analysis. I. Segmentation and surface reconstruction. Neuroimage 9:179-194.

Efron B, Tibshirani R (1993) An introduction to the bootstrap. London: Chapman and Hall.

Ekstrom LB, Roelfsema PR, Arsenault JT, Bonmassar G, Vanduffel W (2008) Bottom-up dependent gating of frontal signals in early visual cortex. Science 321:414-417.

Felleman DJ, Van Essen DC (1991) Distributed hierarchical processing in the primate cerebral cortex. Cereb Cortex 1:1-47.

Fize D, Vanduffel W, Nelissen K, Denys K, Chef d'Hotel C, Faugeras O, Orban GA (2003) The retinotopic organization of primate dorsal V4 and surrounding areas: a functional magnetic resonance imaging study in awake monkeys. J Neurosci 23:7395-7406.

Friston KJ, Holmes AP, Worsley KJ, Poline JB, Frith C, Frackowiak RSJ (1995) Statistical parametric maps in functional imaging: a general linear approach. Hum Brain Mapp 2:189-210.

Ghazanfar AA, Nicolelis MA (1997) Nonlinear processing of tactile information in the thalamocortical loop. J Neurophysiol 78:506-510.

Griswold MA, Jakob PM, Heidemann RM, Nittka M, Jellus V, Wang J, Kiefer B, Haase A (2002) Generalized autocalibrating partially parallel acquisitions (GRAPPA). Magn Reson Med 47:1202-1210.

Grosbras MH, Paus T (2002) Transcranial magnetic stimulation of the human frontal eye field: effects on visual perception and attention. J Cogn Neurosci 14:1109-1120.

Hamker FH (2005) The reentry hypothesis: the putative interaction of the frontal eye field, ventrolateral prefrontal cortex, and areas V4, IT for attention and eye movement. Cereb Cortex 15:431-447.

Handy TC, Kingstone A, Mangun GR (1996) Spatial distribution of visual attention: perceptual sensitivity and response latency. Percept Psychophys 58:613-627.

Hawkins HL, Hillyard SA, Luck SJ, Mouloua M, Downing CJ, Woodward DP (1990) Visual attention modulates signal detectability. J Exp Psychol Hum Percept Perform 16:802-811.

Huerta MF, Krubitzer LA, Kaas JH (1987) Frontal eye field as defined by intracortical microstimulation in squirrel monkeys, owl monkeys, and macaque monkeys. II. Cortical connections. J Comp Neurol 265:332-361.

Kastner S, Ungerleider LG (2000) Mechanisms of visual attention in the human cortex. Annu Rev Neurosci 23:315-341.

Kastner S, De Weerd P, Desimone R, Ungerleider LG (1998) Mechanisms of directed attention in the human extrastriate cortex as revealed by functional MRI. Science 282:108-111.

Kolster H, Mandeville JB, Arsenault JT, Ekstrom LB, Wald LL, Vanduffel W (2009) Visual field map clusters in macaque extrastriate visual cortex. J Neurosci 29:7031-7039.

Lee J, Maunsell JH (2009) A normalization model of attentional modulation of single unit responses. PLoS ONE 4:e4651.

Leite FP, Tsao D, Vanduffel W, Fize D, Sasaki Y, Wald LL, Dale AM, Kwong KK, Orban GA, Rosen BR, Tootell RB, Mandeville JB (2002) Repeated fMRI using iron oxide contrast agent in awake, behaving macaques at 3 tesla. Neuroimage 16:283-294.

Li X, Lu ZL, Tjan BS, Dosher BA, Chu W (2008) Blood oxygenation leveldependent contrast response functions identify mechanisms of covert attention in early visual areas. Proc Natl Acad Sci U S A 105:6202-6207.

Logothetis NK (2008) What we can do and what we cannot do with fMRI. Nature 453:869-878.

Luck SJ, Chelazzi L, Hillyard SA, Desimone R (1997) Neural mechanisms of spatial selective attention in areas $\mathrm{V} 1, \mathrm{~V} 2$, and $\mathrm{V} 4$ of macaque visual cortex. J Neurophysiol 77:24-42.

Mandeville JB, Jenkins BG, Chen YC, Choi JK, Kim YR, Belen D, Liu C, Kosofsky BE, Marota JJ (2004) Exogenous contrast agent improves sensitivity of gradient-echo functional magnetic resonance imaging at $9.4 \mathrm{~T}$. Magn Reson Med 52:1272-1281.

Martínez-Trujillo J, Treue S (2002) Attentional modulation strength in cortical area MT depends on stimulus contrast. Neuron 35:365-370.

McNicol D (1972) A primer of signal detection theory. London: George Allen and Unwin. 
Michelson AA (1927) Studies in optics. Chicago: University of Chicago.

Mioche L, Singer W (1988) Long-term recordings and receptive field measurements from single units of the visual cortex of awake unrestrained kittens. J Neurosci Methods 26:83-94.

Moeller S, Freiwald WA, Tsao DY (2008) Patches with links: a unified system for processing faces in the macaque temporal lobe. Science 320:1355-1359.

Moore CI, Nelson SB, Sur M (1999) Dynamics of neuronal processing in rat somatosensory cortex. Trends Neurosci 22:513-520.

Moore T, Armstrong KM (2003) Selective gating of visual signals by microstimulation of frontal cortex. Nature 421:370-373.

Moore T, Fallah M (2004) Microstimulation of the frontal eye field and its effects on covert spatial attention. J Neurophysiol 91:152-162.

Moore T, Armstrong KM, Fallah M (2003) Visuomotor origins of covert spatial attention. Neuron 40:671-683.

Müller HJ, Humphreys GW (1991) Luminance-increment detection: capacitylimited or not? J Exp Psychol Hum Percept Perform 17:107-124.

Naka KI, Rushton WA (1966) S-potentials from colour units in the retina of fish (Cyprinidae). J Physiol 185:536-555.

Nelissen K, Vanduffel W, Orban GA (2006) Charting the lower superior temporal region, a new motion-sensitive region in monkey superior temporal sulcus. J Neurosci 26:5929-5947.

Palmer C, Cheng SY, Seidemann E (2007) Linking neuronal and behavioral performance in a reaction-time visual detection task. J Neurosci 27:8122-8137.

Reynolds JH, Chelazzi L (2004) Attentional modulation of visual processing. Annu Rev Neurosci 27:611-647.

Reynolds JH, Heeger DJ (2009) The normalization model of attention. Neuron 61:168-185.

Reynolds JH, Pasternak T, Desimone R (2000) Attention increases sensitivity of V4 neurons. Neuron 26:703-714.

Rizzolatti G, Riggio L, Dascola I, Umiltá C (1987) Reorienting attention across the horizontal and vertical meridians: evidence in favor of a premotor theory of attention. Neuropsychologia 25:31-40.

Ruff CC, Blankenburg F, Bjoertomt O, Bestmann S, Freeman E, Haynes JD, Rees G, Josephs O, Deichmann R, Driver J (2006) Concurrent TMSfMRI and psychophysics reveal frontal influences on human retinotopic visual cortex. Curr Biol 16:1479-1488.

Schall JD, Morel A, King DJ, Bullier J (1995) Topography of visual cortex connections with frontal eye field in macaque: convergence and segregation of processing streams. J Neurosci 15:4464-4487.

Schwabe L, Obermayer K, Angelucci A, Bressloff PC (2006) The role of feedback in shaping the extra-classical receptive field of cortical neurons: a recurrent network model. J Neurosci 26:9117-9129.

Sclar G, Maunsell JH, Lennie P (1990) Coding of image contrast in central visual pathways of the macaque monkey. Vision Res 30:1-10.
Silvanto J, Lavie N, Walsh V (2006) Stimulation of the human frontal eye fields modulates sensitivity of extrastriate visual cortex. J Neurophysiol 96:941-945.

Simons DJ (1985) Temporal and spatial integration in the rat SI vibrissa cortex. J Neurophysiol 54:615-635.

Somers DC, Todorov EV, Siapas AG, Toth LJ, Kim DS, Sur M (1998) A local circuit approach to understanding integration of long-range inputs in primary visual cortex. Cereb Cortex 8:204-217.

Stanton GB, Bruce CJ, Goldberg ME (1995) Topography of projections to posterior cortical areas from the macaque frontal eye fields. J Comp Neurol 353:291-305.

Taylor PC, Nobre AC, Rushworth MF (2007) FEF TMS affects visual cortical activity. Cereb Cortex 17:391-399.

Tolias AS, Sultan F, Augath M, Oeltermann A, Tehovnik EJ, Schiller PH, Logothetis NK (2005) Mapping cortical activity elicited with electrical microstimulation using FMRI in the macaque. Neuron 48:901-911.

Tootell RB, Reppas JB, Kwong KK, Malach R, Born RT, Brady TJ, Rosen BR, Belliveau JW (1995) Functional analysis of human MT and related visual cortical areas using magnetic resonance imaging. J Neurosci 15:3215-3230

Ungerleider LG, Desimone R (1986a) Projections to the superior temporal sulcus from the central and peripheral field representations of V1 and V2. J Comp Neurol 248:147-163.

Ungerleider LG, Desimone R (1986b) Cortical connections of visual area MT in the macaque. J Comp Neurol 248:190-222.

Vanduffel W, Fize D, Mandeville JB, Nelissen K, Van Hecke P, Rosen BR, Tootell RB, Orban GA (2001) Visual motion processing investigated using contrast agent-enhanced fMRI in awake behaving monkeys. Neuron 32:565-577.

Vanduffel W, Fize D, Peuskens H, Denys K, Sunaert S, Todd JT, Orban GA (2002) Extracting 3D from motion: differences in human and monkey intraparietal cortex. Science 298:413-415.

Van Essen DC, Drury HA, Dickson J, Harwell J, Hanlon D, Anderson CH (2001) An integrated software suite for surface-based analyses of cerebral cortex. J Am Med Inform Assoc 8:443-459.

Vogels R, Orban GA (1994) Activity of inferior temporal neurons during orientation discrimination with successively presented gratings. J Neurophysiol 71:1428-1451.

Wardak C, Ibos G, Duhamel JR, Olivier E (2006) Contribution of the monkey frontal eye field to covert visual attention. J Neurosci 26:4228 -4235.

Williford T, Maunsell JH (2006) Effects of spatial attention on contrast response functions in macaque area V4. J Neurophysiol 96:40-54.

Worsley KJ, Marrett S, Neelin P, Vandal AC, Friston KJ, Evans AC (1996) A unified statistical approach for determining significant signals in images of cerebral activation. Hum Brain Mapp 4:58-73. 